\title{
Introduction to the 2020 (HICSS-53) Digital Government and the Internet of Things (IoT) Minitrack
}

\author{
Hans J. Scholl \\ The Information School \\ University of Washington \\ jscholl@uw.edu
}

\author{
John C. Bertot \\ College of Information Studies \\ University of Maryland College \\ Park \\ jbertot@umd.edu
}

\author{
Euripidis N. Loukis \\ Department of Information and \\ Communication Systems \\ Engineering \\ University of the Aegean \\ eloukis@aegean.gr
}

The Internet of Things (IoT) is an observably disruptive technology and a fundamental game changer already impacting almost all aspects of everyday individual and organizational life. IoT is massively changing the way humans act and interact with each other even without noticing or knowing IoT's underlying workings.

IoT embodies and enacts the idea of connecting everyday physical and logical objects that intelligently interact with each other in various including autonomous ways via the Internet. IoT integrates, is enabled by, or enables other advanced technologies such as Big Data Analysis, Blockchain, Artificial Intelligence, and Machine Learning. Application areas include but are not limited to autonomous driving and navigation, one's personal home, traditional cars, and lifestyle, as well as basically all industries such as energy, transportation, manufacturing, retail, logistics, agriculture, and healthcare among others.

Furthermore, governments at all levels and in all branches are beginning to implement IoT (smart cities, smart infrastructures, safety/security, etc). IoT technologies are rapidly permeating all layers and aspects of any existing infrastructures fundamentally improving, changing, extending, and redefining them.

While the potential benefits of IoT will be significant, IoT also poses never-encountered vulnerabilities and opportunities for harmful impacts. A deep understanding of the respective challenges and opportunities and their evolution over time already has moved into the focus of legislators and rule makers around the world. Effective regulatory regimes and legislation, which without stifling facilitate the IoT-based evolution, are also subject of this minitramck.

The purpose of this minitrack is to explore the role of government, and, in particular, Digital Government, in the evolution and proliferation of the Internet of Things.

Submissions to this minitrack were invited from various disciplines and backgrounds, both technical and non-technical, for example,

- Government roles in advancing certain IoT network architectures and designs
- Public sector smart objects and smart object connections

- Public sector IoT data flows and data analytics

- IoT security in government

- IoT and open data

- IoT management platform in the public sector

- IoT-related regulation and statutes

- Smart grids (transportation, energy, safety, etc.)

- IoT social impacts and outcomes

- Ethical considerations, privacy concerns, and the right to know

- Artificial intelligence and IoT in government

This brand new minitrack received only a few submissions in its inaugural year, one of which was accepted. Since this topic will undoubtedly attract an increasing number of submissions in the future, we decided to not transfer the first accepted paper to another minitrack, but rather make a statement that we believe in the growing relevance and importance of this topic.

In the next editions of the DG Track at HICSS-54 and HICSS-55, we will maintain our commitment to organizing and holding this particular minitrack.

The inaugural paper of this minitrack is entitled "Here, there, but not everywhere: Adoption and diffusion of IoT in Swedish municipalities." It is coauthored by Ted Saarikko, Ulrika Westergren, Katrin Jonsson. At the municipal level it studies the decision making regarding implementation and diffusion of IoT in local governments. The study describes the incentives and barriers of IoT adoption giving a glimpse of the drivers for future IoT adoption in local public sectors. 